\title{
Intérêt des racines transformées pour le maintien du pouvoir pathogène de quelques agents cryptogamiques
}

\author{
PM Molot *, JP Leroux, M Conus, A Burgerjeon-Glandard \\ INRA, station de pathologie végétale, domaine Saint-Maurice, BP 94, 84140 Montfavet, France
}

(Reçu le 29 octobre 1989; accepté le 26 décembre 1989)

\begin{abstract}
Résumé - Trois champignons dont le pouvoir pathogène en culture artificielle ne se maintient pas de façon satisfaisante, ont été expérimentés : Pyrenochaeta lycopersici, Phytophthora infestans et Rhizoctonia violacea. Sauf pour $P$ lycopersici, la conservation sous azote liquide est impossible ou très aléatoire; les techniques de maintien en condition anaérobie ne conviennent guère (et encore avec des réserves) qu'à $P$ infestans. Pour les 3 parasites, nous avons utilisé avec succès la culture associée du champignon et de racines transformées. Cette technique permet, dans la majorité des cas, un léger gain d'agressivité. Plusieurs cultures de racines transformées ont été expérimentées : tomate, pomme de terre, carotte, betterave à sucre et endive. Après passages répétés sur ces cultures, nous n'avons observé ni préférence, ni adaptation parasitaire. L'intérêt des cultures associées en phytopathologie est discuté.
\end{abstract}

Pyrenochaeta lycopersici / Phytophthora infestans / Rhizoctonia violacea / tomate / pomme de terre / carotte / betterave à sucre / endive

Summary - Use of transformed roots to preserve the pathogenicity of fungal cultures. Alternative means were sought to preserve 3 fungi, Pyrenochaeta lycopersici (tab I), Phytophthora infestans (tab II) and Rhizoctonia violacea (tab III) the pathogenicity of which is not maintained satisfactorily in artificial culture. With the exception of $\mathrm{P}$ lycopersici, preservation in liquid nitrogen is either not possible or very uncertain; maintenance in anaerobic conditions may suit $\mathrm{P}$ infestans only if at all. We successfully maintained the 3 pathogens in culture on transformed roots; with this method, the aggressiveness of the strains is maintained, and nearly always slightly increased, and it is not necessary to inoculate and reisolate from host plants in the greenhouse. Several cultures of transformed roots have been tested: tomato, potato, carrot, sugar-beet and endive. After repeated transfers on these cultures, no parasitic specialization was observed. The interest of mixed cultures is discussed.

Pyrenochaeta lycopersici / Phytophthora infestans / Rhizoctonia violacea / tomato / potato / carrot / sugarbeet / endive

\section{INTRODUCTION}

Pour les phytopathologistes, le maintien en bonne condition d'une collection d'agents cryptogamiques en culture artificielle soulève des problèmes dont la solution n'est pas toujours aisée : - choix d'un milieu favorable à la croissance mycélienne ou à la fructification du champignon (les 2 milieux étant souvent différents) ; - détermination des conditions optimales de développement, notamment température, qualité de la lumière, photopériode, etc. II convient également de s'assurer que la souche est totalement indem- ne de pollutions (certaines bactéries étrangères pouvant s'exprimer sur un milieu et pas sur un autre).

Conserver une souche nécessite en outre, dès que celle-ci commence à vieillir, des repiquages dont la fréquence peut, dans certains cas, être très élevée (toutes les 3 semaines par exemple). Or de nombreuses observations ont montré qu'après plusieurs repiquages successifs, beaucoup de champignons perdaient fréquemment une partie, sinon la totalité de leur pouvoir pathogène. Pour $y$ apporter un remède, il importe donc de réduire, voire même de supprimer les repi-

\footnotetext{
* Correspondance et tirés à part
} 
quages grâce à des techniques spéciales : utilisation du froid (azote liquide ou lyophilisation), mise des cultures sous eau ou sous huile. Ces techniques ne sont malheureusement pas applicables à tous les champignons.

Si l'on choisit de repiquer régulièrement une souche, il est toujours possible de lui redonner son pouvoir pathogène initial en effectuant une contamination artificielle sur une plante hôte et en pratiquant un nouvel isolement de la souche. Mais cette technique est longue et sujette à des risques de pollution, donc de perte de souche, surtout si l'on a à faire à des parasites racinaires (Molot, 1973).

Dans ce dernier cas, une solution élégante peut être trouvée dans l'emploi des racines transformées. En utilisant des hairy roots, autrement dit des racines transformées par Agrobacterium rhizogenes, il est commode de cultiver in vitro des racines et ce en l'absence de la bactérie (Tepfer et Tempe, 1981) : les racines induites par $A$ rhizogenes sont génétiquement transformées, car la bactérie intègre de manière durable, dans le génome des cellules qu'elle infecte, un fragment de son ADN, le T-DNA, porté par un plasmide pathogène Ri (Chilton et al, 1982).

In vitro, les racines transformées se caractérisent par de nombreuses ramifications latérales, l'abondance de leurs poils absorbants et leur tendance à pousser horizontalement. Elles se prêtent facilement à des études in vitro, en modèle réduit, de la rhizosphère.

Leur utilisation dans l'étude des symbiotes obligatoires et dans la compréhension des processus d'infection a déjà fait l'objet d'applications intéressantes. Les endomycorhizes à vésicules et arbuscules (champignons $V A$ ) établissent, avec les racines de la plupart des espèces végétales, une association symbiotique vitale. Leur culture pure est impossible, mais en revanche l'association in vitro entre des racines transformées de Calystegia sepium et 2 endomycorhizes VA, Glomus mosseae et Gigaspora margarita, a été réalisée avec succès (Mugnier et Mosse, 1987 ; Bécard et Fortin, 1988). De plus, d'autres champignons, parasites obligatoires de racines, peuvent être étudiés de façon précise grâce aux hairy roots : Plasmodiophora brassicae, agent causal de la hernie des Crucifères (Mugnier, 1987a) et Polymyxa betae, vecteur du Beet Necrotic Yellow Vein Virus (BNYVV) responsable de la rhizomanie de la betterave (Yacoub, 1987 ; Mugnier, 1987a). Enfin, grâce au système des cultures bicompatimentées, une meilleure approche des relations hôte-parasite sur cultures associées de racines transformées et de champignons du sol, a été entreprise : Phytophthora,
Pythium, Fusarium, Rhizoctonia (Mugnier, 1987b)

Dans le cadre de cette étude, nous nous proposons de cultiver sur racines transformées de diverses plantes, quelques parasites dont le pouvoir pathogène apparaît, après plusieurs repiquages sur milieux classiques, assez instable. Nous avons utilisé 2 champignons racinaires: Pyrenochaeta lycopersici, agent du corky root de la tomate et Rhizoctonia violacea, responsable de dépérissements chez de nombreuses plantes, ainsi qu'un parasite plutôt inféodé aux parties aériennes: Phytophthora infestans, agent du mildiou de plusieurs Solanacées. Nous porterons principalement notre attention sur l'évolution du pouvoir pathogène de ce matériel fongique.

\section{MATÉRIEL ET MÉTHODES}

\section{Matériel végétal}

Nous avons expérimenté sur 3 agents cryptogamiques phytopathogènes : Pyrenochaeta lycopersici (9 souches), Phytophthora infestans (5 souches), Rhizoctonia violacea ( 2 souches). Les caractéristiques de chacune de ces souches sont décrites dans le tableau I.

Afin de tester le pouvoir pathogène, nous avons utilisé pour :

- Pyrenochaeta lycopersici : des racines de jeunes tomates (var. Monalbo);

- Phytophthora infestans : des feuilles en survie ou des plantes entières de tomate (var. Monalbo) ou de pomme de terre (var. Bintje). II s'agit alors de plantes élevées en serre et âgées de 4 semaines ;

- Rhizoctonia violacea : des racines de carotte (var. Touchon), des racines de betterave à sucre (ITB $n^{\circ} 3368$ ), des tubercules de pomme de terre (var. Bintje).

Dans ce cas, carottes et pommes de terre proviennent de plantes à maturité, tandis que les racines de betterave sont prélevées sur des plantes élevées en serre et âgées de 2 mois.

\section{Culture de racines transformées}

Nous avons utilisé différentes cultures de racines transformées correspondant aux plantes suivantes : carotte, pomme de terre, betterave, tomate, endive. Ces cultures nous ont été aimablement communiquées par M Mugnier de la société Rhône-Poulenc Agrochimie. Elles sont conservées sur milieu liquide à l'obscurité et sans agitation. Au moment de leur utilisation, on les transfère sur un milieu gélosé de même composition ne renfermant pas d'hormone (tableau II).

Lorsque la culture de racines atteint dans la boîte de Petri un diamètre voisin de $5 \mathrm{~cm}$, on dépose à la surface des tissus un ou plusieurs implants provenant d'une culture mycélienne classique sur milieu nutritif gélosé. A partir de ces implants se développent des colonies fongiques strictement inféodées à la croissan- 
Tableau I. Caractéristiques des différentes souches.

\begin{tabular}{|c|c|c|c|c|}
\hline & & & & \\
\hline & Souche & Année & Plante & Lieu \\
\hline & 7 & 1982 & Tomate & Montfavet (84) \\
\hline & 57 & 1985 & Tomate & Canaries (Espagne) \\
\hline & 59 & 1986 & Tomate & Montfavet (84) \\
\hline Pyrenochaeta & 60 & 1985 & Salade & Montfavet (84) \\
\hline lycopersici & 60 bis & 1985 & Tomate & Montfavet (84) \\
\hline & & 1985 & Melon & Les Vignères (84) \\
\hline & 64 & 1985 & Salade & Montfavet (84) \\
\hline & 68 & 1986 & Tomate & Montfavet (84) \\
\hline & 72 & 1987 & Tomate & Eyragues (13) \\
\hline & LQ 2 & 1983 & Tomate & Bouches-du-Rhône (13) \\
\hline Phytophthora & $\mathrm{RP}$ & 1985 & Tomate & \\
\hline infestans & SL & 1983 & Tomate & Sainte-Livrade (47) \\
\hline & 855 & 1981 & Tomate & \\
\hline & VIL & 1984 & Tomate & Lot $(46)$ \\
\hline $\begin{array}{l}\text { Rhizoctonia } \\
\text { violacea }\end{array}$ & $\begin{array}{l}\text { VN } 78 \\
\text { DR } 80\end{array}$ & $\begin{array}{l}1978 \\
1980\end{array}$ & $\begin{array}{l}\text { Asperge } \\
\text { Asperge }\end{array}$ & $\begin{array}{l}\text { Vaison-la-Romaine (84) } \\
\text { Nyons }(26)\end{array}$ \\
\hline
\end{tabular}

Tableau II. Composition du milieu adapté à la culture de racines transformées.

\begin{tabular}{|c|c|c|}
\hline \multicolumn{3}{|c|}{ Macro-éléments de Murashige et Skoog ( $\mathrm{mg} / \mathrm{L})$} \\
\hline $\begin{array}{l}\mathrm{NO}_{3} \mathrm{NH}_{4} \\
\mathrm{KNO}_{3} \\
\mathrm{Ca} \mathrm{Cl}_{2} 6 \mathrm{H}_{2} \mathrm{O} \\
\mathrm{Mg} \mathrm{SO}_{4} 7 \mathrm{H}_{2} \mathrm{O} \\
\mathrm{KH}_{2} \mathrm{PO}_{4} \\
\mathrm{Na}_{2} \mathrm{EDTA} \\
\mathrm{Fe} \mathrm{SO}_{4} 7 \mathrm{H}_{2} \mathrm{O}\end{array}$ & $\begin{array}{c}1650 \\
1900 \\
440 \\
370 \\
17 \\
37,3 \\
27,8\end{array}$ & $\begin{array}{l}\text { Nitrate d'ammonium } \\
\text { Nitrate de potassium } \\
\text { Chlorure de calcium } \\
\text { Sulfate de magnésium } \\
\text { Phosphate monopotassique } \\
\text { EDTA sel disodique } \\
\text { Sulfate ferreux }\end{array}$ \\
\hline \multicolumn{3}{|c|}{ Micro-éléments de Murashige et Skoog $(\mathrm{mg} / \mathrm{L})$} \\
\hline 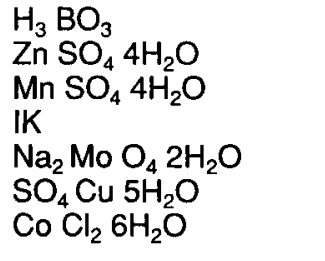 & $\begin{array}{c}6,2 \\
8,6 \\
22,6 \\
0,83 \\
0,35 \\
0,025 \\
0,025\end{array}$ & $\begin{array}{l}\text { Acide borique } \\
\text { Sulfate de zinc } \\
\text { Sulfate de manganèse } \\
\text { lodure de potassium } \\
\text { Molybdate de sodium } \\
\text { Sulfate de cuivre } \\
\text { Chlorure de cobalt }\end{array}$ \\
\hline \multicolumn{3}{|c|}{ Vitamines de Morel $(\mathrm{mg} / \mathrm{L})$} \\
\hline $\begin{array}{l}\text { Calcium pantothénate } \\
\text { méso-Inositol } \\
\text { Biotine } \\
\text { Acide nicotinique } \\
\text { Pyridoxine } \mathrm{HCl} \\
\text { Thiamine } \mathrm{HCl}\end{array}$ & $\begin{array}{c}1 \\
100 \\
0,01 \\
1 \\
1 \\
1\end{array}$ & \\
\hline $\begin{array}{l}\text { Acide indolbutyrique } \\
\text { Saccharose } \\
\text { AGAR } \\
\text { pH } 6,5\end{array}$ & $\begin{array}{c}1 \mathrm{mg} / \mathrm{L} \\
30 \mathrm{~g} / \mathrm{L} \\
8 \mathrm{~g} / \mathrm{L}\end{array}$ & \\
\hline
\end{tabular}

ce racinaire (le milieu pour les cultures de racines ne convenant généralement pas aux champignons). Au bout de 15 jours de confrontation, on repique, après découpage à l'emporte-pièce, des implants (racines + champignon) sur une culture vierge de racines. Plusieurs repiquages successifs sur racines d'un même hôte peuvent ainsi être pratiqués (dans certains cas, jusqu'à 6).

\section{Contrôle du pouvoir pathogène}

Après plusieurs repiquages sur milieu classique ou sur racines transformées, l'agressivité des différentes souches est appréciée par des tests de laboratoire ou de serre. 


\section{Au laboratoire}

Pyrenochaeta lycopersici : des implants mycéliens calibrés sont mis au contact de jeunes racines de tomate issues directement d'une germination in vitro selon une technique décrite par ailleurs (Clerjeau et Conus, 1973). La lecture des symptômes s'effectue selon le barème suivant :

-0 = rien;

-1 = nécrose sous implant;

- 2 = nécrose commençant à dépasser l'implant;

$-3=$ nécrose plus longue demeurant marron clair;

-4 = nécrose nette, brun foncé, partant des 2 côtés de l'implant.

Phytophthora infestans : des folioles de l'étage moyen, prélevées sur des tomates âgées de 4 semaines, sont mises en survie sur de l'eau, en boîte de Petri. Au centre du limbe et sur une légère blessure, on dépose un implant calibré d'une culture mycélienne $(\varphi=0,4 \mathrm{~cm})$ à partir duquel se développe une nécrose tissulaire dont on mesure le diamètre.

Rhizoctonia violacea: des tubercules de pomme de terre, des racines de carotte ainsi que des racines de betterave (âgées de 2 mois) sont désinfectés superficiellement à l'alcool. A leur surface et sans pratiquer de blessure, on dépose un implant calibré de culture mycélienne $(\varphi=0,6 \mathrm{~cm})$. Les hyphes se développent en donnant une colonie circulaire dont on mesure le diamètre.

\section{En serre ( $P$ infestans uniquement)}

Sur des plantes âgées de 4 semaines (pomme de terre ou tomate), on pulvérise une suspension de sporanges ajustée pour chaque souche à $10000 / \mathrm{mL}$. L'incubation est réalisée en chambre humide à $18^{\circ} \mathrm{C}$ sous un éclairage faible. L'appréciation des symptômes s'effectue en attribuant à chaque plante une note visuelle globale allant de 0 (pas de maladie) à 5 (mortalité).

\section{RÉSULTATS}

\section{Pyrenochaeta lycopersici (Tableau III)}

Sur 9 souches expérimentées, nous constatons qu'après repiquage sur milieu classique, toutes ont perdu leur agressivité. En revanche, 6 passages successifs sur racines transformées la maintiennent à un bon niveau. Par rapport au test de contrôle de départ, 4 souches $(7,57,60$ bis, 62) se révèlent légèrement plus agressives, tandis que 2 autres $(68,72)$ semblent un peu moins pathogènes. Remarquons que les racines d'endive, expérimentées sur 2 souches seulement, sembleraient capables, au même titre que les racines de tomate, de conserver les potentialités du matériel cryptogamique. Parallèlement à ces essais, nous avons pu, après conservation pendant 6 mois sans repiquage à $4{ }^{\circ} \mathrm{C}$, freiner les pertes d'agressivité qu'il est courant d'observer en conditions classiques.

\section{Phytophthora infestans (Tableau IV)}

Le pouvoir pathogène des implants mycéliens obtenus après repiquage soit sur milieu normal (avoine, pois chiche), soit sur racines transformées (tomate ou pomme de terre) a été contrôlé simultanément sur tomate et pomme de terre. On remarque, pour les 5 souches étudiées, qu'après passages répétés sur racines transformées, il y a une nette augmentation d'agressivité. Cette hausse s'observe surtout sur feuilles en survie,

Tableau III. Evolution de l'agressivité de 11 souches de Pyrenochaeta lycopersici en fonction du mode de repiquage. L'agressivité est exprimée par rapport à de jeunes racines de tomate (note de 0 à 4). - = absence de mesures.

\section{Au bout de 6 mois}

Numéro Au départ des sources
Après repiquage sur :

Racines
transformées de

Sans repiquage (conservation $+4^{\circ} \mathrm{C}$ )

Milieu
classique

\begin{tabular}{rlllll}
\hline 7 & & & & & 0 \\
57 & 0,8 & 0 & 1,3 & - & 2,6 \\
59 & 3,2 & 0 & 4 & - & 2,4 \\
60 & 3 & 0 & 0 & - & 0,6 \\
60 bis & 0,8 & 0 & 0,6 & 1,1 & 0,7 \\
62 & 1,2 & 0 & 1,5 & - & 2,3 \\
64 & 0,9 & 0 & 2,4 & - & 2,9 \\
68 & 2,3 & 0 & 2,4 & - & 1,8 \\
72 & 2,9 & 0 & 1,6 & 2,2 & \\
\hline
\end{tabular}


sans doute à cause de la plus grande sensibilité du test. Les résultats ne montrent pas de différence entre racines transformées de tomate ou de pomme de terre.

\section{Rhizoctonia violacea (Tableau V)}

La souche VN 78 est plus agressive que la souche DR 80 et, d'une façon générale, la sensibilité des plantes hôtes dans l'ordre décroissant est la suivante : carotte, betterave, pomme de terre. Comme pour les 2 champignons précédents et quelle que soit la souche considérée, il apparaît qu'après passages sur racines transformées, la souche augmente d'agressivité par rapport à la même souche repiquée sur milieu classique. Cependant, il ne semble pas qu'après passage sur racines transformées, le champi- gnon acquiert une spécificité parasitaire. Par exemple, après passage sur racines de carotte, son agressivité se trouve augmentée, mais aussi bien sur carotte que sur d'autres plantes (pomme de terre ou betterave à sucre).

\section{DISCUSSION - CONCLUSION}

En dépit du caractère assez restreint de notre étude faisant intervenir 3 champignons, chacun d'eux ayant été repiqué 6 fois sur diverses racines transformées, nous avons pu constater que, mis à part quelques souches, il y avait dans la grande majorité des cas, non seulement maintien de l'agressivité, mais encore assez souvent une augmentation de cette dernière. Confronté au problème de vieillissement des souches, il apparaît donc tout à fait possible d'éviter ainsi un

Tableau IV. Influence de 2 modes de repiquage (sur racines transformées ou sur milieu classique) sur le pouvoir pathogène de 5 souches de Phytophthora infestans vis-à-vis de la tomate (var. Monalbo) ou de la pomme de terre (var. Bintje). Sur feuilles en survie, on mesure le diamètre des nécroses en mm. Sur plantes entières, la sensibilité est exprimées par une note de 0 à 5.

Six passages sur racines transformées de:

Tomate
Six repiquages sur milieu classique (avoine, pois-chiche)

$\begin{array}{lllllllllllllllllll}\text { Plantehôte testée } & \text { LQ2 } & \text { RP } & \text { SL } & 855 & \text { VIL } & \text { Moy } & \text { LQ2 } & \text { RP } & \text { SL } & 855 & \text { VIL } & \text { Moy } & \text { LQ2 } & \text { RP } & \text { SL } & 855 & \text { SL } & \text { Moy }\end{array}$

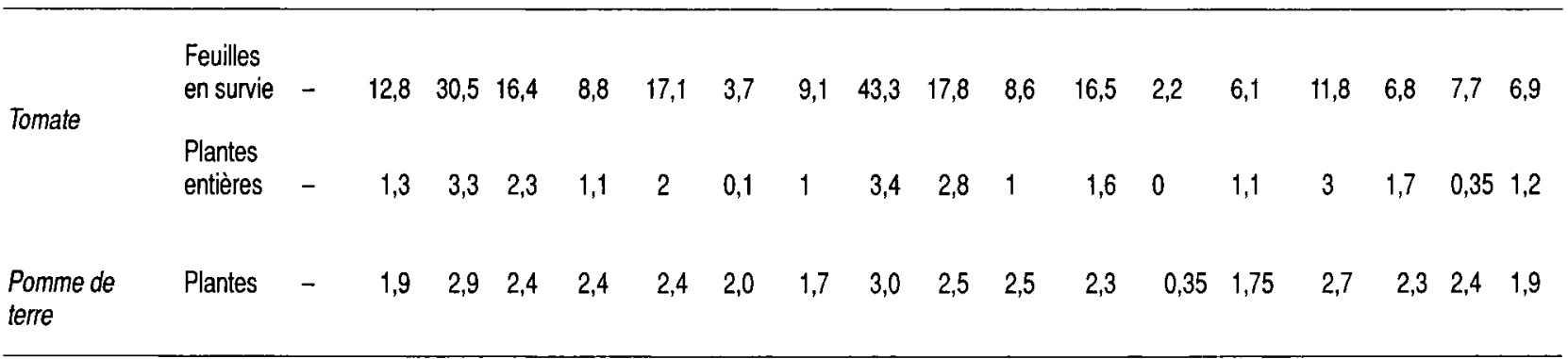

Tableau V. Influence de 2 modes de repiquage (sur racines transformées ou sur milieu classique) sur les contaminations artificielles par 2 souches de Rhizoctonia violacea. Les croissances mycéliennes sur racines (carotte, betterave) ou tubercules (pomme de terre) sont exprimées en $\mathrm{mm}$. $\mathrm{A}=$ souche $\mathrm{DR} 80$ de $R$ violacea; $\mathrm{B}=$ souche $\mathrm{VN} 78$ de $R$ violacea.

Six passages sur racines transformées de :

Six repiquages sur milieu classique

\begin{tabular}{lrrrrrrrrrrr}
$\begin{array}{l}\text { Plante hôte } \\
\text { testée }\end{array}$ & \multicolumn{3}{c}{ Carotte } & \multicolumn{3}{c}{ Pomme de terre } & \multicolumn{2}{c}{ Betterave } & \multicolumn{3}{c}{ Tomate } \\
& A & B & A & B & A & B & A & B & A & B \\
\hline \multirow{2}{*}{ Carotte } & 30,0 & 29,5 & 25,6 & $3 n, 3$ & 21,1 & 29,6 & 25,7 & 27 & 22,6 & 23,9 \\
Pomme de terre & 3,2 & 4,9 & 3,7 & 4,1 & 3,2 & 4,7 & 5,0 & 5,4 & 2,5 & 4,2 \\
Betterave à sucre & 14,7 & 17,9 & 18,1 & 19,9 & 16,2 & 16,4 & 17,3 & 19,4 & 12,7 & 14,7 \\
\hline
\end{tabular}


«repassage» sur l'hôte, en conditions généralement non stériles, opération toujours délicate, surtout lorsqu'il s'agit d'un parasite racinaire.

A l'image des racines normales, les racines transformées sont le lieu de synthèse de nombreux métabolites secondaires, notamment d'alcaloïdes tropaniques (Hammil et al, 1986). On comprend dès lors qu'elles soient propices à la croissance mycélienne de nombreux champignons. Cependant, le fait qu'elles soient incapables de synthétiser les substances produites par les parties aériennes et aussi qu'elles soient 100 à 1000 fois plus sensibles aux auxines (Shen et al, 1988) que les racines normales, explique peut-être que nous n'ayons jamais observé d'adaptation parasitaire après passages répétés sur racines transformées d'un hôte donné.

Malgré les avantages offerts (notamment gain d'agressivité), les repiquages sur racines transformées représentent une technique lourde qu'il convient de ne réserver qu'à quelques cas d'espèces, chaque fois que le recours à des techniques plus simples est impossible. A ce propos, nous avons résumé dans le tableau $\mathrm{VI}$, le comportement des 3 champignons étudiés après séjour dans l'azote liquide ou après immersion sous eau stérile.

Les observations recueillies ici montrent que si la culture sur racines transformées est inutile avec Pyrenochaeta lycopersici que l'on conserve très bien dans l'azote liquide, en revanche elle peut offrir des avantages substantiels avec $R h i$ zoctonia violacea ou Phytophthora infestans. Cette étude mériterait d'être poursuivie avec d'autres champignons.

\section{RÉFÉRENCES}

Becard G, Fortin JA (1988) Early events of vesiculararbuscular mycorrhiza formation on $\mathrm{Ri}$ T-DNA transformed roots. New Phytol 108, 2, 211-218

Clerjeau M, Conus M (1973) Méthode rapide de contamination de jeunes plantules de tomates par Pyrenochaeta lycopersici Schneider et Gerlach. Ann Phytopathol 5, 2, 143-150

Chilton MD, Tepfer DA, Petit A, David C, Casse-Delbart F, Tempe J (1982) Agrobacterium rhizogenes inserts T-DNA into the genome of the host plant root cells. Nature 295, 432-434

Hammil JD, Parr AJ, Robins RJ, Rhodes MJC (1986) Secondary product formation by cultures of Beta vulgaris and Nicotiana rustica transformed with Agrobacterium rhizogenes. Plant Cell Rep 5, 111114

Molot PM (1973) Mise au point sur Rhizoctonia violacea. Biologie du parasite, technique d'inoculation artificielle, possibilités de lutte. CR $4^{e}$ réunion Eucarpia Asperge, Versailles, 77-88

Mugnier J (1987a) Infection by Polymyxa betae and Plasmodiophora brassicae of roots containing rootinducing transferred DNA of Agrobacterium rhizogenes. Phytopathology 77, 4, 539-542

Mugnier J (1987b) Spécificité d'hôte entre Rhizoctonia solani et des racines transformées par Agrobacterium rhizogenes. SFP, $32^{\mathrm{e}}$ colloque 14-15 Mai, Angers

Mugnier J, Mosse B (1987) Vesicular arbuscular mycorrhizal infection in transformed root-inducing T-DNA roots grown axenically. Phytopathology 77 , 7, 1 045-1 050

Shen W, Petit A, Guerin J, Tempé J (1988) Hairy roots are more sensitive to auxin than normal roots. Proc Natl Acad. Sci USA 85, 3 417-3 421

Tepfer D, Tempe J (1981) Production d'agropine par des racines formées sous l'action d'Agrobacterium rhizogenes, souche A4. CR Acad Sci 292, série III, 153-156

Yacoub A (1987) Culture in vitro de Polymyxa betae en association avec des racines de betterave transformées par Agrobacterium rhizogenes. Thèse de doctorat, INA, Paris

Tableau VI. Comportement des 3 champignons étudiés après séjour dans l'azote liquide ou après immersion sous eau stérile. + = repart; $-=$ ne repart pas; $\sim$ = repart (selon la souche); ? = manipulation non réalisée.

\section{Refroidissement brutal}

\section{Refroidissement \\ ménagé \\ + glycérol}
1 mois
6 mois

Pyrenochaeta

lycopersici

Phytophthora

infestans

Rhizoctonia

violacea

$\begin{array}{cccc}+ & + & ? & ? \\ - & - & + & \sim \\ \sim & + & - & -\end{array}$

